\title{
Biological Agent Serovar Name
}

National Cancer Institute

\section{Source}

National Cancer Institute. Biological Agent Serovar Name. NCI Thesaurus. Code C158304.

The serovar name of the biological challenge agent. 